psychiatrist versus SOS-PD assessments in 14 patients. The sensitivity was $90.1 \%$ (95\% CI 75.6-97.9\%) and positive predictive value $96.8 \%$ (95\% CI 90.5-100). The specificity was $96.7 \%$ (95\% CI 82.7-99.4) and the negative predictive value $90.6 \%$ (95\% CI 80.5-100). The ICC of 16-paired observations was 0.90 (95\% CI 82.7-99.4) for the SOS-PD scale.

Conclusions The SOS-PD scale shows promising validity for screening PD. Future research is aimed to establish the psychometric properties in a multicenter study.

\section{0-160 DEVELOPMENT OF A PARENT'S GUIDE FOR NEWBORNS WITH HYPOXIC-ISCHAEMIC ENCEPHALOPATHY (HIE) AND THERAPEUTIC HYPOTHERMIA (TH)}

${ }^{1} \mathrm{~N}$ Herranz, ${ }^{2} \mathrm{~V}$ Violant, ${ }^{3} \mathrm{~A}$ Balaguer, ${ }^{4} \mathrm{R}$ Del Rio, ${ }^{5} \mathrm{D}$ Estrada, ${ }^{4} \mathrm{~A}$ Garcia-Alix. ${ }^{1}$ Division of Neonatology, Hospital Sant Joan de Deu, Barcelona, Spain; ${ }^{2}$ Didactica y Organización Educativa, Universidad de Barcelona, Barcelona, Spain; ${ }^{3}$ Facultad of Medicine, Universidad Internacional de Cataluña, Barcelona, Spain; ${ }^{4}$ Division of Neonatology, Hospital Sant Joan de Deu, Barcelona, Spain, ${ }^{5}$ Agència de Qualitat I Avaluació Sanitàrias de Catalunya, Agència de Qualitat I Avaluació Sanitàrias de Catalunya, Barcelona, Spain

\subsection{6/archdischild-2014-307384.227}

Backgrounds $\mathrm{TH}$ is the only available treatment today for infants with HIE and it is considered the standard of care. Being a parent of a baby with HIE treated with $\mathrm{TH}$ in a neonatal intensive care is a highly traumatic event.

Aims To develop an explicative guide for parents of newborns with HIE receiving TH based on parent's needs.

Methods To identify significant issues for parents, a descriptive qualitative study was designed. Study population were parents of newborns with HIE who received TH. Convenience sampling was used, stratified according to the severity of HIE. Two focus groups with 16 parents of newborns with moderate and mild HIE, respectively were performed. Twenty pictures were taken reflecting dimensions and categories extracted from bibliographic review. These images were used to encourage parents discourse. Interviews were recorded prior to parental consent and later transcribed. Inductive content analysis was performed using ATLAS. TI-V.6.2.

Results Three themes emerged: HIE information, cooling treatment and coping strategies for parents. The guide developed followed this structure and contents were divided into 16 meaningful questions and a glossary. The final parents guide had a didactic format with simple language, drawings, and parents verbatims. It is annexed to a Spanish clinical practice guide (CPG) on HIE.

Conclusions Qualitative results highlight the importance for parents to receive good information about HIE, cooling, and coping strategies. This guide is a tool that could improve family coping during hospitalisation of newborns with $\mathrm{HIE}$ and $\mathrm{TH}$. Incorporating parents perspectives in the CPG increase its quality.

\section{0-161 CEREBRAL OXYGENATION BEFORE AND AFTER NEUROSURGICAL PLACEMENT OF A VENTRICULAR RESERVOIR IN NEONATES WITH PHVD}

AJ Brouwer, T Alderliesten, LS de Vries, PMA Lemmers. Neonatology, Wilhelmina Children's Hospital University Medical Center Utrecht, Utrecht, Netherlands

10.1136/archdischild-2014-307384.228
Background There is no consensus regarding optimal timing in treating posthaemorrhagic ventricular dilatation (PHVD). NearInfraRed Spectroscopy (NIRS) is a non invasive method, measuring cerebral regional oxygenation $\left(\mathrm{rScO}_{2}\right) \cdot \mathrm{rScO}_{2}$ values below $40-45 \%$ might be associated with cerebral ischaemia.

Hypothesis: $\mathrm{rScO}_{2}$ can provide additional information about cerebral oxygenation in infants with PHVD and may therefore be of value to determine timing of intervention.

Methods We measured $\mathrm{rcSO}_{2}$ in 13 neonates before and after neurosurgical placement of a ventricular reservoir. Based on ventricular index (VI; Levene), distinction was made in neonates treated early (VI $<$ p97 $+4 \mathrm{~mm}$ ) and those treated late for their PHVD (VI $\geq$ p $97+4 \mathrm{~mm}$ ).

Results Median GA 31 wks (27-37 wks) and median BW 1750 g (1145-3270 g). Five neonates were treated early and 8 late. In the early intervention group, pre-, and postoperative $\mathrm{rScO} 2$ values were comparable (median 52\%, 45-58\% IQR vs 57\%, 44$60 \%$ IQR). Preoperative $\mathrm{rScO}_{2}$ was lower in the late intervention group compared to postoperative values (median 33\%, 2643\% IQR vs 47\%, 39-49\% IQR).

In 7 late intervention infants $\mathrm{rScO}_{2}$ was $<45 \%$ preoperatively, so at risk for cerebral ischemia, In $2 \mathrm{rScO}_{2}$ remained $<45 \%$ postoperatively.

Conclusions Neonates with $\mathrm{VI} \geq 97+4 \mathrm{~mm}$ do have a compromised cerebral oxygenation, and usually react to cerebrospinal fluid drainage with recovery of the $\mathrm{rScO}_{2}$ values to within the normal range. Infants in the early intervention group were within normal range pre- and postoperatively. NIRS might be of additional clinical value in progressive PHVD in order to determine optimal timing for intervention.

\section{0-162 CLINICAL IMPLICATIONS OF MRI-PROCEDURE IN PRETERM NEONATES}

M Bouman, K Keunen, LS De Vries, F Groenendaal, MJNL Benders, AJ Brouwer. Wilhelmina Childrens Hospital, UMC Utrecht, Utrecht, Netherlands

\subsection{6/archdischild-2014-307384.229}

Background and aim Magnetic Resonance Imaging (MRI) of the brain at 30 weeks Postmenstrual age (PMA) is part of routine care for preterms born $<28$ weeks gestational age (GA), because of their high risk of brain injury.

The aim was to evaluate fluctuations in vital parameters following the MRI procedure.

Methods and patients We compared clinical parameters in 30 infants $4 \mathrm{~h}$ before and after the MRI: number of apneas and bradycardias, changes in oxygen requirement, respiratory support, and rectal temperature. Oral chloralhydrate sedation $(30-50 \mathrm{mg} /$ $\mathrm{kg}$ ) was administered upon discretion of the attending neonatologist.

Results Infants had the following clinical characteristics: mean GA 26 + 4 wks (24-28 wks), mean BW 1012 g (610-1520 g), PMA at scan was $30+6$ wks $(29+6-31+6$ wks $)$ with weight of $1397 \mathrm{~g}$ (980-1860 g). Infants $<1500 \mathrm{~g}$, were transported in an MRI-compatible incubator (26/30). 12/30 (40\%) infants were sedated with chloralhydrate.

None of the infants had $>4$ apneas or $>3$ bradycardias before the MRI. After the MRI 5 infants (all unsedated) had $>4$ apneas (5-7 apneas), 2 infants (1 sedated) had $>3$ bradycardias (5-6). After the MRI, $\mathrm{FiO}_{2}$ was increased in $10 / 30$ patients, max change 0.06 , more respiratory support was needed in 5 infants (3 sedated): PEEP from +4 to PEEP $+5 \mathrm{cmH}_{2} \mathrm{O}$, and 
temperature was lower in $20 / 30$ patients, max decrease $1,3^{\circ} \mathrm{C}$ (before $37.1^{\circ} \mathrm{C}\left(36.3-37.9^{\circ} \mathrm{C}\right)$ vs. $36.6^{\circ} \mathrm{C}\left(35.9-37.4^{\circ} \mathrm{C}\right)$ ).

Conclusions Early MRI scanning using an MRI incubator is a relatively safe procedure in clinically stable infants. Use of sedation was not associated with clinically relevant changes, although these findings warrant further investigation.

\section{0-163 DOES EARLY SCREENING LEAD TO HIGHER PREVALENCE OF PAEDIATRIC DELIRIUM?}

E Ista, $\mathrm{H}$ te Beest, M de Hoog, D Tibboel, M van Dijk. Intensive Care Unit, Erasmus University Medical Center - Sophia Children's Hospital, Rotterdam, Netherlands

\subsection{6/archdischild-2014-307384.230}

Introduction Early screening of paediatric delirium (PD) allows for early intervention if necessary. The aim of this study was to determine if early screening with the SOS-PD scale led to higher prevalence of PD in ICU patients.

Methods A prospective before-after study design was applied in a population of children aged $>3$ months and admitted for $\geq 48$ $\mathrm{h}$ to the PICU. In the before-period the prevalence of PD was estimated in terms of the number of children with PD confirmed by the consulting psychiatrist. During the after-period nurses systematically assessed the children with the SOS-PD scale three times a day in addition to the psychiatric consultation (SOS-PD score $\geq 4$ ).

Results 148 and 150 children were included in the before and after period, respectively. The prevalence of PD was $6.1 \%$ and $8.7 \%$ for the before and after period respectively (see Table). The relative risk of PD with early screening was 1:43 (95\% CI 0.63 to 3.23 ). In 33 patients (22\%) the SOS-PD score was $\geq 4$ on one or more occasions. In 14 of these patients, the child psychiatrist was consulted. In the remaining patients the child psychiatrist was not consulted for the following reasons: only once a high score $(n=9)$, adverse effects of sedatives $(n=4)$, and underlying disease/motor restlessness $(n=6)$.

Abstract 0-163 Table 1 Patient characteristics and prevalence of PD

\begin{tabular}{l|c|c|c}
\hline & $\begin{array}{c}\text { Before period - usual } \\
\text { care }(\mathrm{N}=148)\end{array}$ & $\begin{array}{c}\text { After period - early } \\
\text { screening PD }(\mathrm{N}=150)\end{array}$ & p-value \\
\hline Gender (F/M) & $59 / 89$ & $76 / 74$ & 0.06 \\
\hline Age (months) & $37(15-124)$ & $54(14-146)$ & 0.22 \\
\hline Length of stay ICU (days) & $7(4-13)$ & $6(4-11)$ & 0.11 \\
\hline Prevalence of PD & $9(6.1 \%)$ & $13(8.7 \%)$ & 0.40 \\
\hline $\begin{array}{l}\text { Number of assessments per } \\
\text { child* }\end{array}$ & - & $9(4-19)$ & - \\
\hline
\end{tabular}

Conclusions Systematic early screening of PD resulted in a higher incidence of PD and could contribute to timely start of treatment.

\section{Allergology}

\section{0-164 SPECIFIC IMMUNOGLOBULIN E TO ARA H 2 AS PREDICTOR FOR PEANUT ALLERGY IN CHILDREN IN A GENERAL DUTCH HOSPITAL}

${ }^{1} \mathrm{M}$ Schots, ${ }^{2} \mathrm{AC}$ de Mol, ${ }^{2} \mathrm{YM}$ Roosen, ${ }^{3} \mathrm{HJ}$ Vermeer, ${ }^{2} \mathrm{AW}$ Vriesman. ${ }^{1}$ Paediatrics, VU Medical Center, Amsterdam, Netherlands; ${ }^{2}$ Paediatrics, Albert Schweitzer Hospital, Dordrecht, Netherlands; ${ }^{3}$ Clinical Chemistry, Albert Schweitzer Hospital, Dordrecht, Netherlands

10.1136/archdischild-2014-307384.231
Background Specific immunoglobulin E (sIgE) to Ara h 2 is described as a potential factor for diagnosing peanut allergy in children. However for the Dutch children, limited data are available. In this study the diagnostic value of sIgE to Ara h2 for children in a general non-university hospital is evaluated and compared with the existing data.

Methods Data from 137 peanut sensitised children were collected retrospectively. The primary outcome was peanut allergy or tolerance confirmed by food challenges. Different possible predictors, including sIgE to Ara h $2(\mathrm{n}=52)$, were identified by multivariate backward stepwise logistic regression analysis. All significant predictors were combined in a formula for prediction of peanut allergy. Different essential cut-off points were obtained by an ROC curve.

Results Multivariate analysis resulted in $\operatorname{sgE}$ to Ara h 2 as only predictor for peanut allergy, with a discriminative ability of 0.87 (95\% CI, 0.77-0.97). Sensitivity and specificity values of respectively 55\% and 95\% were found at a sIgE to Ara h 2 cut-off value of $4.25 \mathrm{kU} / \mathrm{L}$. Hundred percent specificity was reached at a cut-off point of $5.61 \mathrm{kU} / \mathrm{L}$. The mean (SD) sIgE to Ara h 2 level for allergic children was $21.49 \mathrm{kU} / \mathrm{L}$ (SD 30.65) compared to $1.07 \mathrm{kU} / \mathrm{L}(1.56)$ for tolerant children ( $\mathrm{p}=0.001)$.

Conclusions Specific IgE to Ara h 2 is the best predictor for peanut allergy in sensitised children in a non-university hospital, comparable to previously published data. These results are a step forward to a generalisation to the Dutch children population.

\section{0-165 PRENATAL EXPOSURE TO ENDOCRINE DISRUPTING CHEMICALS (EDCS) IS RELATED TO ALLERGIC SYMPTOMS IN 12 MONTH OLD CHILDREN}

${ }^{1}$ I Quaak, ${ }^{1} \mathrm{LC}$ Roosendaal, ${ }^{1} \mathrm{M}$ De Cock, ${ }^{2} \mathrm{MH}$ Lamoree, ${ }^{1} \mathrm{M}$ Van de Bor. ${ }^{1}$ Health and Life Sciences, VU University, Amsterdam, Netherlands; ${ }^{2}$ Institute for Environmental Studies, VU University, Amsterdam, Netherlands

\subsection{6/archdischild-2014-307384.232}

Background In daily life, we are exposed to thousands of manmade chemicals. Some of these chemicals do have endocrine disrupting properties. Prenatal exposure to EDCs may interrupt the maturation of the immune system and lead to childhood allergies.

Objective To determine the relationship between prenatal exposure to EDCs and allergic symptoms in 12 month old children.

Methods Fourty-two pregnant women were recruited at the first antenatal visit to the midwife. Exposure to four different classes of EDCs was determined in cord blood and breast milk: perfluorinated alkyl acids, PCBs, organochlorine pesticides and phthalate metabolites. Allergic symptoms at the age of 12 months was assessed by means of questionnaires. Gender, maternal BMI, parental education and parity were taken into account as possible confounders. Logistic regression analyses were carried out.

Results A significant positive relation was found between prenatal PFOS exposure and allergic symptoms in children at the age of 12 months (OR 4.84; $\mathrm{p}=0.04$ ). In addition, prenatal exposure to PFOA and MECPP was positively related to allergic symptoms in 12 month old children, while a negative association was found for phthalate metabolites MEHHP and MEOHP. However, these associations were not significant (Table 1).

Discussion Prenatal exposure to PFOS is significantly related to allergic symptoms at the age of 12 months. Moreover, associations between exposure to several other EDCs and allergic symptoms in children of 12 months old have been found. In 